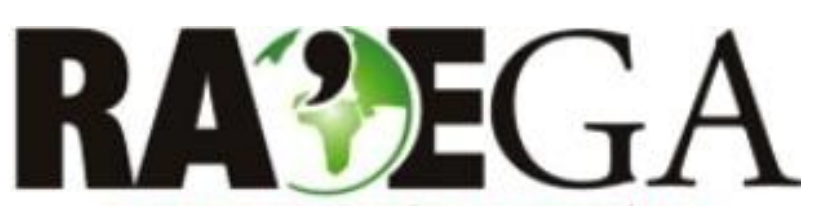

O ESPACYO GEOGRÁFICO EM ANÁLISE

\title{
USO DO NDVI NA ANÁLISE DA ESTRUTURA DA VEGETAÇÃO E EFETIVIDADE DA PROTEÇÃO DE UNIDADE DE CONSERVAÇÃO NO CERRADO
}

\section{USE OF NDVI IN ANALYSIS OF STRUCTURE VEGETATION AND PROTECTION EFFECTIVENESS OF CONSERVATION UNIT IN THE CERRADO}

\author{
Roberto Macedo Gamarra \\ Universidade Federal de Mato Grosso do Sul - UFMS \\ Campo Grande, MS, Brasil \\ e-mail: rmgamarra@gmail.com \\ Mara Cristina Teixeira-Gamarra \\ Universidade Federal de Mato Grosso do Sul - UFMS \\ Campo Grande, MS, Brasil \\ e-mail: mara.c.teixeira@gmail.com

\begin{abstract}
Martha Gilka Gutiérrez Carrijo Secretaria Estadual de Meio Ambiente, Planejamento, Ciência e Tecnologia Instituto de Meio Ambiente de Mato Grosso do Sul - IMASUL

Costa Rica, MS, Brasil e-mail: imapcostarica@yahoo.com.br
\end{abstract} \\ Antonio Conceição Paranhos Filho \\ Universidade Federal de Mato Grosso do Sul - UFMS \\ Campo Grande, MS, Brasil \\ e-mail: antonio.paranhos@pq.cnpq.br
}

\section{Resumo}

Diferentes índices de vegetação podem ser utilizados para o estudo e avaliação da vegetação. O NDVI é utilizado com sucesso para classificar a distribuição global de vegetação, inferir variabilidades ecológicas e ambientais, produção de fitomassa, radiação fotossintética ativa e a produtividade de culturas. Nesse contexto, a utilização do NDVI como medida indireta de 
complexidade e heterogeneidade ambiental pode ser uma abordagem interessante. Assim, o objetivo do presente trabalho foi avaliar a dinâmica da paisagem e a efetividade da proteção de uma unidade de conservação de proteção integral no Cerrado, utilizando geotecnologias. Foram coletados em campo dados de estrutura da vegetação, a cobertura por espécies lenhosas (árvores e arbustos) e a cobertura de serapilheira de 12 fragmentos de vegetação arbórea do Parque Estadual das Nascentes do Rio Taquari (PENRT), que foram comparados com os valores do Índice de Vegetação por Diferença Normalizada (NDVI). A relação entre variáveis de estrutura da vegetação e o índice de vegetação (NDVI) e a análise multitemporal da complexidade e heterogeneidade do habitat do PENRT mostraram-se ferramentas muito eficazes para avaliar a efetividade da proteção oferecida pela unidade de conservação. As geotecnologias contribuem para o estudo da ecologia e conservação da biodiversidade reduzindo tempo e custos na análise ambiental. Os métodos utilizados no presente trabalho poderão ser utilizados em outras regiões do Cerrado que apresentam características semelhantes ao PENRT.

Palavras-chave: Ecologia da paisagem; Geotecnologias; Índice de vegetação; Complexidade do habitat; Heterogeneidade do habitat.

\begin{abstract}
Different vegetation indexes can be used for the study and evaluation of vegetation. The NDVI is successfully used to classify the global distribution of vegetation, infer ecological and environmental variability, biomass production, photosynthetic active radiation and productivity of crops. In this context, NDVI utilization as indirect measure of environmental complexity and heterogeneity can be an interesting approach. The objective of this study was to assess the landscape dynamics and the protection effectiveness of a conservation unit of integral protection in the Cerrado, using geotechnology. Were collected in the field vegetation structure data, the coverage by woody species (trees and shrubs) and the litter cover of 12 fragments of arboreal vegetation of Parque Estadual das Nascentes do Rio Taquari (PENRT), which were compared with the values of Normalized Difference Vegetation Index (NDVI). The relationship between variables of vegetation structure and vegetation index (NDVI) and the multitemporal analysis of complexity and habitat heterogeneity of PENRT proved to be very effective tools to evaluate the effectiveness of the protection offered by the conservation unit. The geothecnologies contribute to the ecology and biodiversity conservation by reducing time and costs in environmental analysis. The methods employed in this study could be used in other Cerrado regions that have similar characteristics to the PENRT.
\end{abstract}

Key-words: Landscape ecology; Geotechnology; Vegetation index; Habitat complexity; Habitat heterogeneity. 
GAMARRA, R. M.; TEIXEIRA-GAMARRA, M. C.; CARRIJO, M. G. G.; PARANHOS FILHO, A. C. USO DO NDVI NA ANÁLISE DA ESTRUTURA DA VEGETAÇÃO E EFETIVIDADE DA PROTEÇÃO DE UNIDADE DE CONSERVAÇÃO NO CERRADO

\section{INTRODUÇÃO}

Nas últimas décadas cerca de $50 \%$ da área original do Cerrado foram convertidas em áreas antropizadas como às atividades agropecuárias, extrativismo mineral, aumento populacional e construção de estradas ou barragens para hidrelétricas (ALHO; MARTINS, 1995, p. 28, MITTERMEIER et al., 1999, p. 35). Este fato é agravado pelo baixo número de áreas protegidas por unidades de conservação, apenas $4,1 \%$ e pela distribuição restrita de muitas espécies da fauna e flora (FELFILI, 2002, p. 60; FELFILI et al., 2002, p. 104).

Apesar disso, o Cerrado é considerado um dos 25 locais do planeta onde estão concentrados $50 \%$ de toda biodiversidade (hotspots), ficando indiscutível a necessidade da conservação de suas espécies e das áreas ocupadas pelo bioma (MITTERMEIER et al., 1999, p. 35). De acordo com o Ministério do Meio Ambiente (MMA, 2007, p. 60), 47,51\% do bioma Cerrado é constituído por Áreas Prioritárias para Conservação, sendo 25,33\% consideradas de importância extremamente alta, 11,72\% de importância muito alta, 9,81\% de importância alta e 0,65\% insuficientemente conhecidas.

A fragmentação de habitats e a conversão da cobertura do solo para fins agrícolas são ameaças constantes para a conservação da biodiversidade no Bioma Cerrado. Estas paisagens dominadas por atividades agrícolas tornaramse mosaicos dinâmicos, que são formados por diferentes usos da terra (CARVALHO et al., 2009, p. 1392). Machado et al. (2004, p. 7) estimam que, o bioma Cerrado deverá ser totalmente destruído no ano de 2030 se as atuais taxas de perda de habitat se manterem.

Nesse contexto, os conceitos da Ecologia da Paisagem aliados às ferramentas/técnicas de Geotecnologias (Sensoriamento Remoto e SIG Sistema de Informações Geográficas) fornecem importante subsídio para o entendimento e planejamento para resolução de problemas ambientais.

A ecologia da paisagem visa estudar a estrutura, função e dinâmica de áreas heterogêneas compostas por ecossistemas interativos, concentrando-se nessas três características fundamentais da paisagem (FORMAN; GODRON, 1986, p. 45; TURNER, 1987, p. 29). A ecologia de paisagem é uma ciência 
básica para o desenvolvimento, manejo, conservação e planejamento da paisagem, ela possibilita que a paisagem seja avaliada sob diversos pontos de vista, permitindo que seus processos ecológicos possam ser estudados em diferentes escalas temporais e espaciais (TURNER, 1987, p. 32).

As geotecnologias representam um dos campos que mais crescem no mundo. Existe uma demanda mundial por profissionais que integrem dados ambientais e imagens de satélite (GEWIN, 2004, p. 376; PARANHOS FILHO et al., 2008, p. 10), demonstrando a importância da utilização desse tipo de ferramenta em análises ambientais. De acordo com Roughgarden et al. (1991, p. 1918) as geotecnologias fornecem à ecologia, dados em larga escala e permitem uma visão de conjunto sobre a estrutura espacial da paisagem.

Diferentes índices de vegetação podem ser utilizados para o estudo e avaliação da vegetação. Entre aqueles já descritos na literatura, há o Índice de Vegetação por Diferença Normalizada (NDVI), que tem relação direta com a fitomassa, permitindo caracterizar alguns aspectos biofísicos da vegetação (PONZONI; SHIMABUKURO, 2007, p. 45). O NDVI é utilizado com sucesso para classificar a distribuição global de vegetação, inferir variabilidades ecológicas e ambientais, produção de fitomassa, radiação fotossintética ativa e a produtividade de culturas (LIU, 2007, p. 132).

Nesse contexto, a utilização do NDVI como medida indireta de complexidade e heterogeneidade ambiental pode ser uma abordagem interessante. A complexidade do habitat pode ser definida como o desenvolvimento do extrato vertical da vegetação juntamente com a densidade de indivíduos (plantas) por unidade de área (ROOT, 1973, p. 116) e a heterogeneidade do habitat é a estrutura da vegetação no plano horizontal, variando de acordo com o hábito das plantas (arbustivo, arbóreo, etc.) presentes na área.

Assim, o objetivo do presente trabalho foi avaliar a dinâmica da paisagem e a efetividade da proteção de uma unidade de conservação de proteção integral no Cerrado, utilizando geotecnologias.

\section{MATERIAIS E MÉTODOS}




\section{1. Área de estudo}

O Parque Estadual das Nascentes do Rio Taquari (PENRT), além de sua importância para o bioma Cerrado, abriga nascentes de importante rio para o Pantanal. Está localizado na região Centro Oeste do Brasil, no Estado de

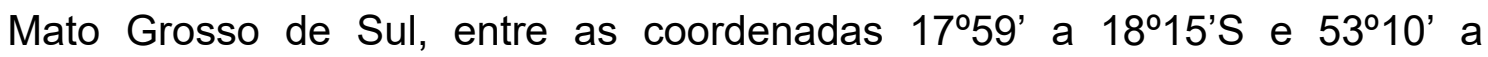
$53^{\circ} 26^{\prime}$ W. O PENRT foi criado pelo Decreto Estadual $n^{\circ} 9.622$ de 09 de outubro de 1999 (MATO GROSSO DO SUL, 1999), com área de 30.618,96 hectares, abrangendo 26.849,62 hectares no Município de Alcinópolis-MS e 3.769,34 hectares em Costa Rica - MS (Figura 1). É uma das áreas núcleo do Corredor de Biodiversidade Emas-Taquari (CONSERVATION INTERNATIONAL DO BRASIL, 2003), ou seja, um dos remanescentes ambientalmente mais importantes no Cerrado, que abriga as nascentes de um importante rio para o Pantanal.

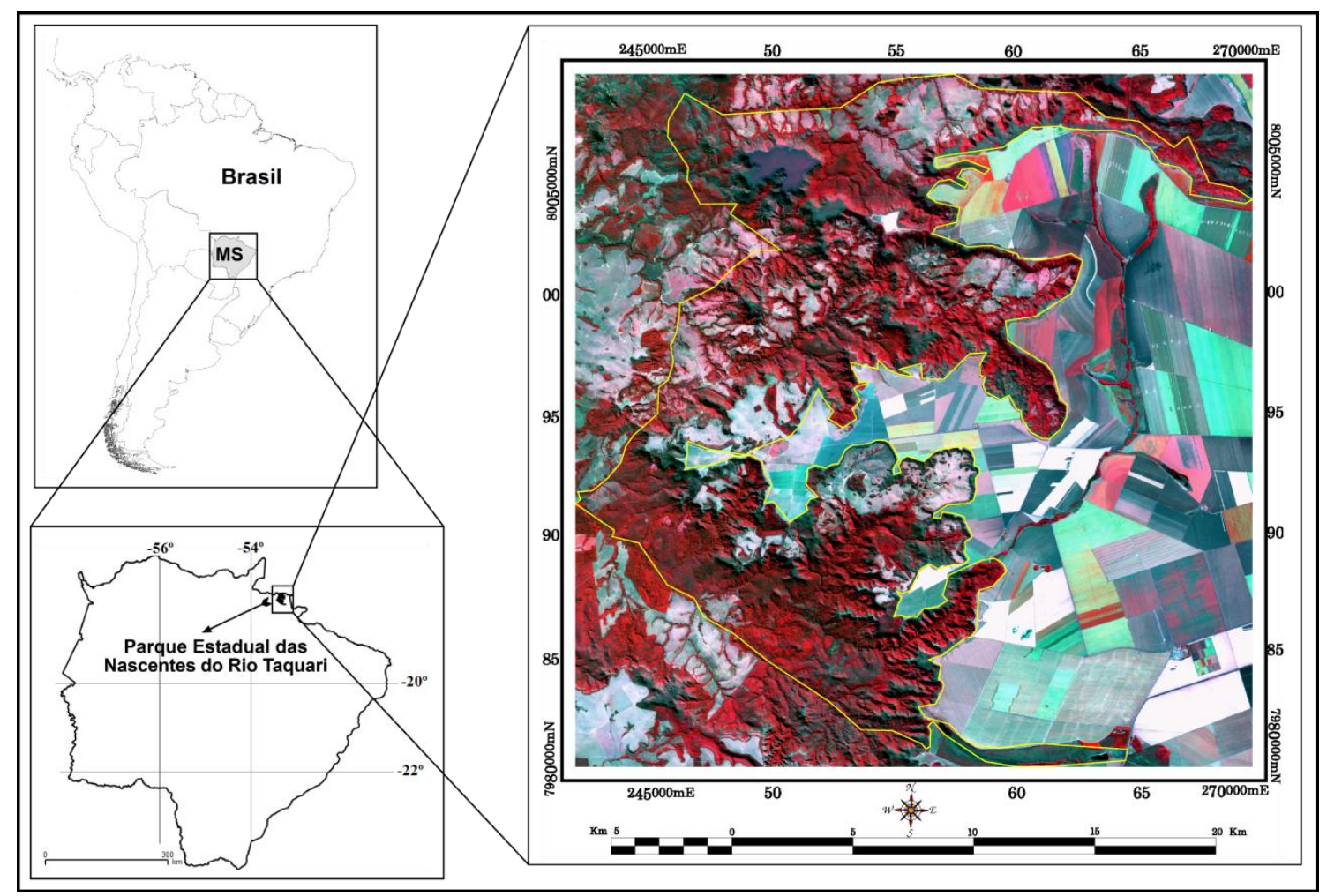

Figura 1. Parque Estadual das Nascentes do Rio Taquari (PENRT). Fonte: Imagem do satélite ALOS sensor AVNIR-2 com resolução espacial de $10 \mathrm{~m}$ de 04 de julho de 2010. Composição falsa cor RGB 432. Limite do PENRT em amarelo. Na porção oeste 
GAMARRA, R. M.; TEIXEIRA-GAMARRA, M. C.; CARRIJO, M. G. G.; PARANHOS FILHO, A. C. USO DO NDVI NA ANÁLISE DA ESTRUTURA DA VEGETAÇÃO E EFETIVIDADE DA PROTEÇÃO DE UNIDADE DE CONSERVAÇÃO NO CERRADO

da imagem, as áreas em vermelho intenso significam vegetação densa. O padrão geométrico na porção leste da imagem mostra áreas agrícolas, que circundam a unidade de conservação.

\subsection{Materiais utilizados}

$\mathrm{Na}$ realização do trabalho foram utilizadas seis cenas do satélite LANDSAT 5 (Land Remote Sensing Satellite), sensor TM (Thematic Mapper), órbita/ponto 224/073, com 30 m de resolução espacial, de 18 de julho de 1984 (INPE, 1984), 16 de julho de 1989 (INPE, 1989), 30 de julho de 1994 (INPE, 1994), 28 de julho de 1999 (INPE, 1999), 25 de julho de 2004 (INPE, 2004) e 26 de julho de 2010 (INPE, 2010). Como material de apoio foram utilizadas ainda duas cenas ortorretificadas do satélite ALOS (Advanced Land Observing Satellite), uma do sensor AVNIR-2 (Advanced Visible and Near Infrared Radiometer type 2) com 10 m de resolução espacial de 04 de julho de 2010 (GLOBALGEO, 2010 a) e outra do sensor PRISM (Panchromatic Remotesensing Instrument for Stereo Mapping) com 2,5 m de resolução espacial de 19 de agosto de 2010 (GLOBALGEO, 2010 b).

Todas as seis cenas do satélite LANDSAT 5 foram georreferenciadas com erro inferior a 1 pixel, utilizando como referência as cenas do satélite ALOS (UTM, fuso 22, WGS84). Utilizou-se também o vetor do limite oficial do PENRT (em formato shapefile) obtido no Sistema Interativo de Suporte ao Licenciamento Ambiental (SISLA) do Instituto de Meio Ambiente de Mato Grosso do Sul (IMASUL) (SISLA, 2008) para recorte dessas cenas.

$\mathrm{Na}$ análise multitemporal foram utilizadas somente imagens LANDSAT, todas da época seca (julho), para garantir maior distinção entre as fitofisionomias e menor interferência da sazonalidade (fenologia). Além de manter as mesmas características da imagem (resolução espacial, espectral e radiométrica).

Foi utilizado o programa Geomatica 9.1 (PCl, 2003) para o georreferenciamento, correção atmosférica, geração do índice de vegetação, recorte e integração de todos os dados em ambiente de Sistema de Informações Geográficas (SIG). 
GAMARRA, R. M.; TEIXEIRA-GAMARRA, M. C.; CARRIJO, M. G. G.; PARANHOS FILHO, A. C. USO DO NDVI NA ANÁLISE DA ESTRUTURA DA VEGETAÇÃO E EFETIVIDADE DA PROTEÇÃO DE UNIDADE DE CONSERVAÇÃO NO CERRADO

\subsection{Coleta de dados em campo}

Foram realizadas três etapas de trabalho de campo, em janeiro de 2009, agosto de 2010 e em dezembro de 2010, no intuito de identificar os tipos de cobertura do solo (incluindo as fitofisionomias descritas por RIBEIRO; WALTER, 1998, p. 89) e fazer o reconhecimento dos fragmentos de vegetação para relacioná-los com as imagens de satélite da área de estudo, utilizando máquina fotográfica digital e receptor GPS de navegação (Global Positioning System).

Também foram coletados dados de estrutura da vegetação como cobertura por espécies lenhosas (árvores e arbustos) utilizando densiômetro esférico e cobertura de serapilheira utilizando grade quadriculada de $1 \mathrm{~m}^{2}$ das fitofisionomias Mata seca e ciliar, Cerradão e Cerrado sentido restrito. Foram amostrados 4 fragmentos de cada fitofisionomia (Mata - 3 fragmentos de Mata seca e um de mata ciliar; Cerradão - 4 fragmentos de Cerradão; e Cerrado - 4 fragmentos de cerrado sentido restrito) com caminhadas aleatórias onde foram realizadas 10 medidas a pelo menos $10 \mathrm{~m}$ de distância uma da outra em cada ponto, totalizando 12 fragmentos amostrados.

Durante o levantamento do histórico da área, os moradores e a gerente do Parque foram entrevistados sobre as mudanças ocorridas na cobertura do solo da área de estudo. Levantamentos bibliográficos também auxiliaram nessa atividade.

\subsection{Estrutura da vegetação e o Índice de Vegetação por Diferença} Normalizada (NDVI)

O Índice de Vegetação por Diferença Normalizada (NDVI) proposto por (ROUSE et al., 1973, p. 309) é calculado pela seguinte relação:

$$
N D V I=(N I R-R) /(N I R+R)
$$

onde,

NIR: Reflectância da vegetação na banda do infravermelho próximo;

R: Reflectância da vegetação na banda do vermelho. 
Para o cálculo do NDVI primeiramente realizou-se a correção atmosférica das seis cenas do satélite LANDSAT 5 através do algoritmo ATCOR2 e após a correção atmosférica foi gerado o NDVI também das seis cenas LANDSAT 5 pela execução do raster calculator utilizando o programa Geomatica 9.1 (PCI, 2003).

Nos mesmos fragmentos onde foram amostradas as variáveis de estrutura da vegetação foram coletadas suas coordenadas. A partir dessas coordenadas foram criados buffers de $100 \mathrm{~m}$ que serviram de limite para 0 recorte dos valores NDVI de 2010, que foram analisados em conjunto com as variáveis de estrutura da vegetação.

Os valores médios do NDVI foram considerados uma medida indireta da complexidade do habitat, pois indicam a complexidade estrutural (vertical) e quantidade de fitomassa do habitat e o desvio padrão dos valores do NDVI foi considerado uma medida indireta da heterogeneidade do habitat, pois indicam a variação/dispersão da complexidade vertical e da fitomassa do ambiente no plano horizontal (CORRÊA et al., 2011, p. 3106; OLIVEIRA et al., 2012, p. 397; GAMARRA, 2013, p. 96).

Foram calculados a média e o desvio padrão do NDVI de 2010, da porcentagem de cobertura lenhosa e da porcentagem de cobertura de serapilheira para cada fragmento amostrado. Para verificar se existe relação entre o NDVI e essas variáveis de estrutura da vegetação separadamente, calculou-se a Regressão Linear Simples no programa BioEstat 5.0 (AYRES et al., 2007).

Também foi calculado a média e o desvio padrão do NDVI de todo o PENRT para os anos de 1984, 1989, 1994, 1999, 2004 e 2010, para avaliar a dinâmica da complexidade e heterogeneidade do habitat do PENRT ao longo do tempo.

\section{RESULTADOS E DISCUSSÃO}

Os valores do índice de vegetação (NDVI), da cobertura vegetal (cobertura por espécies lenhosas - árvores e arbustos) e da cobertura por 
serapilheira para cada fragmento das três fitofisionomias analisadas estão apresentados na Tabela 1.

Tabela 1. Índice de Vegetação por Diferença Normalizada (NDVI), cobertura por espécies lenhosas (árvores e arbustos) e cobertura por serapilheira para cada fragmento de três diferentes fitofisionomias.

\begin{tabular}{|l|c|c|c|c|}
\hline Fitofisionomia & Fragmento & NDVI $^{1}$ & Cobertura Vegetal $^{2}$ & Serapilheira $^{2}$ \\
\hline Mata & 1 & $0,745 \pm 0,036$ & $92,297 \pm 1,396$ & 100 \\
\hline Mata & 2 & $0,831 \pm 0,060$ & $91,554 \pm 2,258$ & 100 \\
\hline Mata & 3 & $0,864 \pm 0,053$ & $92,905 \pm 2,836$ & 100 \\
\hline Mata & 4 & $0,963 \pm 0,069$ & $95,068 \pm 1,236$ & 100 \\
\hline Cerradão & 1 & $0,790 \pm 0,038$ & $88,378 \pm 7,683$ & $95 \pm 15,811$ \\
\hline Cerradão & 2 & $0,774 \pm 0,110$ & $91,351 \pm 3,963$ & $95 \pm 15,811$ \\
\hline Cerradão & 3 & $0,685 \pm 0,086$ & $79,189 \pm 8,622$ & $94 \pm 9,661$ \\
\hline Cerradão & 4 & $0,821 \pm 0,040$ & $89,797 \pm 4,291$ & 100 \\
\hline Cerrado & 1 & $0,468 \pm 0,110$ & $31,893 \pm 23,778$ & $23 \pm 27,101$ \\
\hline Cerrado & 2 & $0,661 \pm 0,155$ & $47,973 \pm 25,715$ & $47 \pm 34,01$ \\
\hline Cerrado & 3 & $0,603 \pm 0,117$ & $76,802 \pm 12,346$ & $65 \pm 16.432$ \\
\hline Cerrado & 4 & $0,560 \pm 0,102$ & $35,811 \pm 16,230$ & $18,3 \pm 12,583$ \\
\hline
\end{tabular}

${ }^{1}$ Valor médio e desvio padrão (Buffer de 100m). ${ }^{2}$ Porcentagem média e desvio padrão.

Para a fitofisionomia Mata verifica-se que os valores do NDVI médio variaram de 0,745 a 0,963, e os valores do desvio padrão do NDVI oscilaram de 0,036 a 0,069. Para Cerradão os valores do NDVI médio oscilaram de 0,685 a 0,821 e o desvio padrão do NDVI variou de 0,038 a 0,110. Já para o Cerrado, os valores do NDVI médio variaram de 0,468 a 0,661 e o desvio padrão do NDVI oscilou de 0,102 a 0,155.

Percebe-se que os maiores valores de complexidade do habitat (NDVI médio) foram registrados na fitofisionomia Mata seguido pelo Cerradão. Verificou-se uma sobreposição de valores de Mata e Cerradão, pois se tratam de dois tipos de formações florestais que apresentam estrutura vertical mais complexa e também maior fitomassa quando comparados com o Cerrado.

Verificou-se também que, os maiores valores de heterogeneidade do habitat (NDVI desvio padrão) foram registrados no Cerrado, pois trata-se da fitofisionomia cerrado sentido restrito, que no PENRT pode variar entre cerrado ralo, cerrado típico e cerrado denso, apresentando diferentes densidades e altura das árvores, ou seja, maior heterogeneidade horizontal. 
O NDVI permite caracterizar parâmetros biofísicos da vegetação, como fitomassa/densidade da vegetação e seu valor é normalizado para o intervalo de -1 a +1 (PONZONI; SHIMABUKURO, 2007, p. 45), sendo que o valor zero se refere aos pixels não vegetados. Esses valores representam uma medida indireta da fitomassa, indicando valores de matéria e energia presentes na área amostrada.

Relacionando os valores do NDVI com parâmetros de estrutura da vegetação registrados em campo percebe-se que houve relação significativamente positiva entre a complexidade do habitat (NDVI médio) e a cobertura por espécies lenhosas (\% média) $\left(r^{2}=0,7179 ; p<0,001\right)$ (Figura 2). Ou seja, a porcentagem média da cobertura por espécies lenhosas explica $71,8 \%$ da variação da complexidade do habitat.

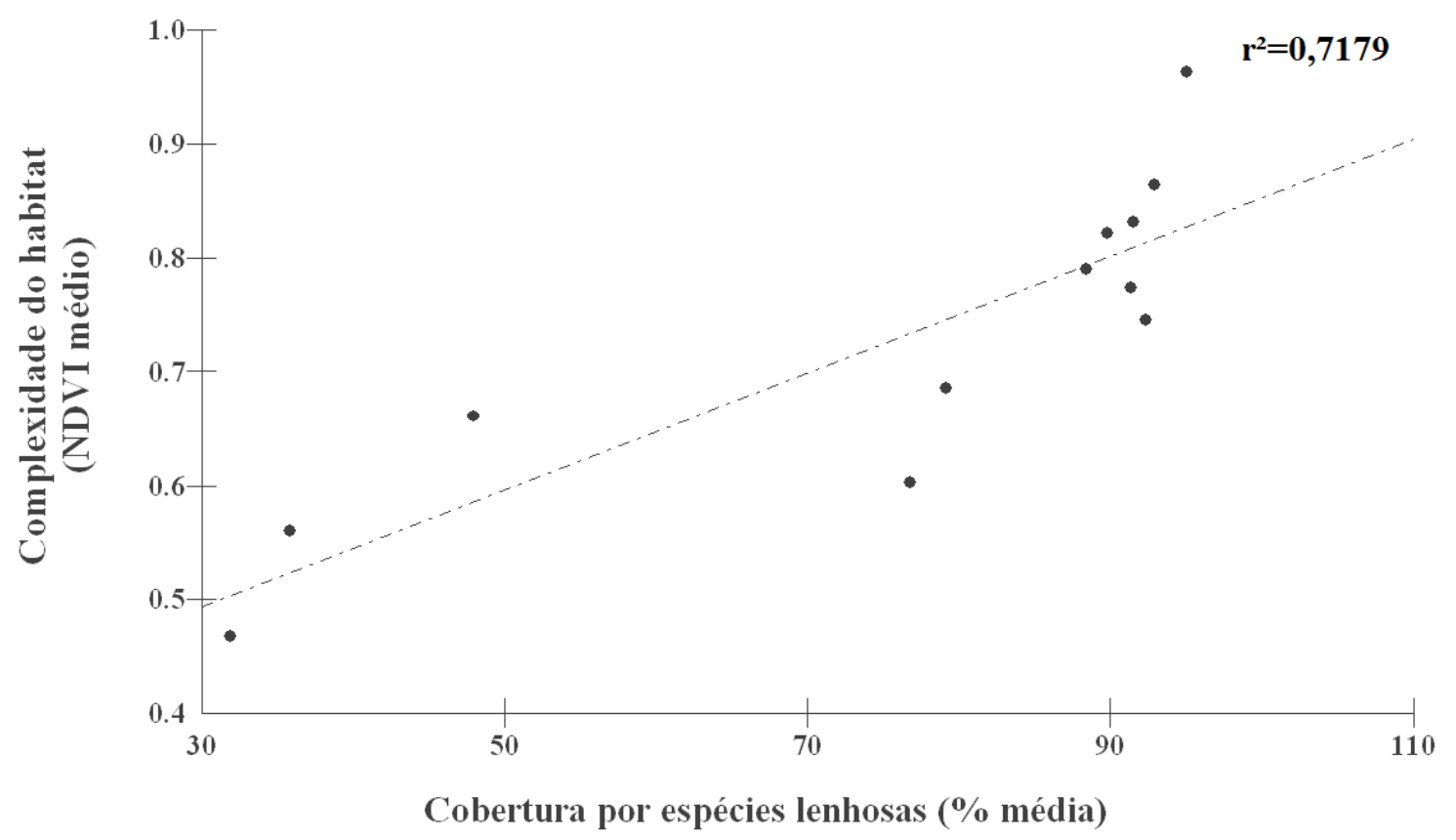

Figura 2. Relação entre complexidade do habitat (NDVI médio) e cobertura por espécies lenhosas (\% média).

Também houve relação significativamente positiva entre a complexidade do habitat (NDVI médio) e a cobertura de serapilheira (\% média) $\left(r^{2}=0,7251\right.$; $\mathrm{p}<0,001$ ) (Figura 3), ou seja, a porcentagem média da cobertura de serapilheira explica $72,5 \%$ da variação da complexidade do habitat. 


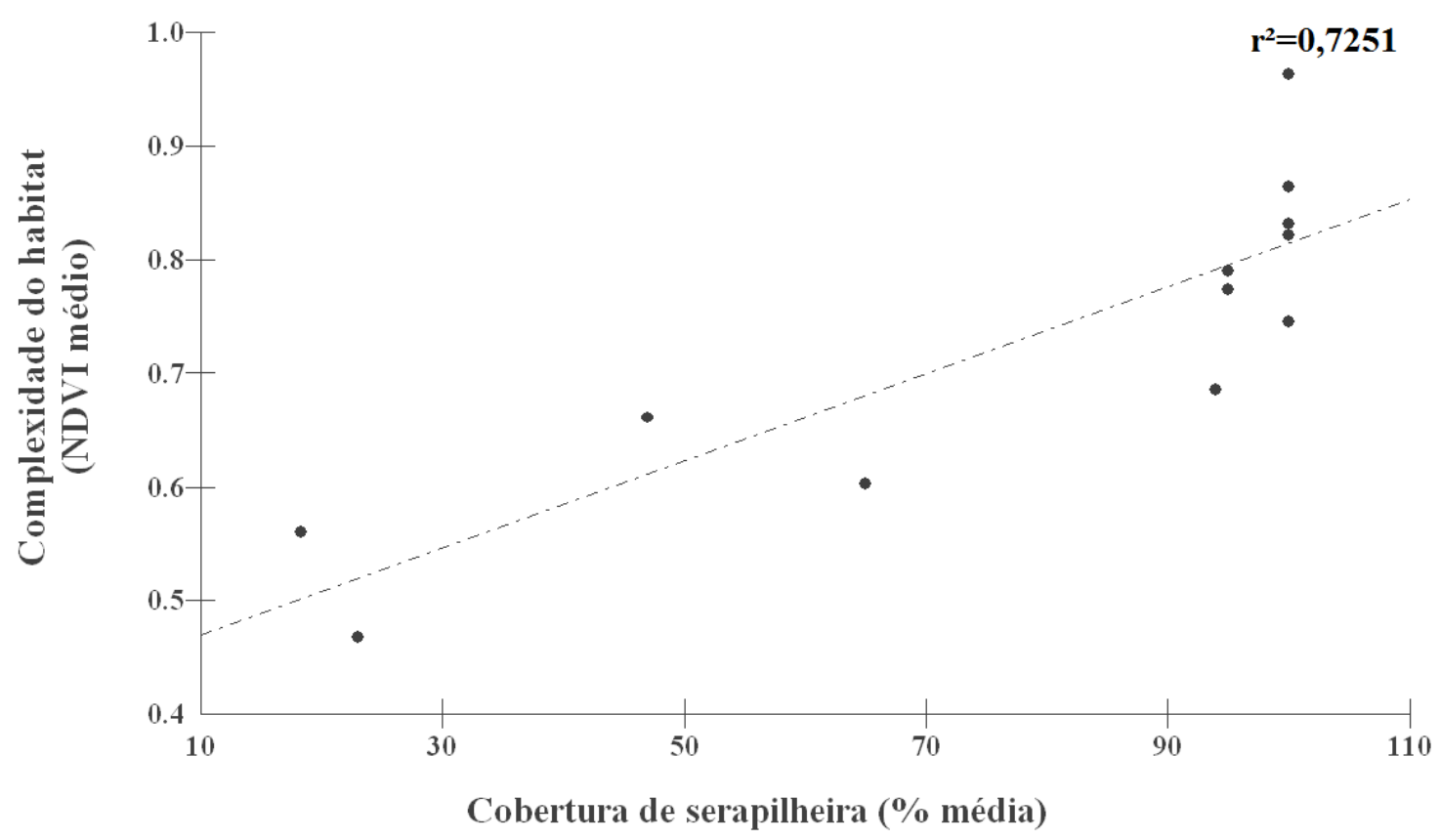

Figura 3. Relação entre complexidade do habitat (NDVI médio) e cobertura de serapilheira (\% média).

Quando analisamos a heterogeneidade do habitat (NDVI desvio padrão) verificou-se relação significativamente positiva com o desvio padrão da cobertura por espécies lenhosas $\left(r^{2}=0,5482 ; p=0,0038\right)$ (Figura 4) e também com o desvio padrão da cobertura de serapilheira $\left(r^{2}=0,6285 ; p=0,0016\right)$ (Figura 5). Assim, a variação da cobertura por espécies lenhosas explica 54,8\% da variação da heterogeneidade do habitat e a variação da cobertura de serapilheira explica $62,9 \%$. 


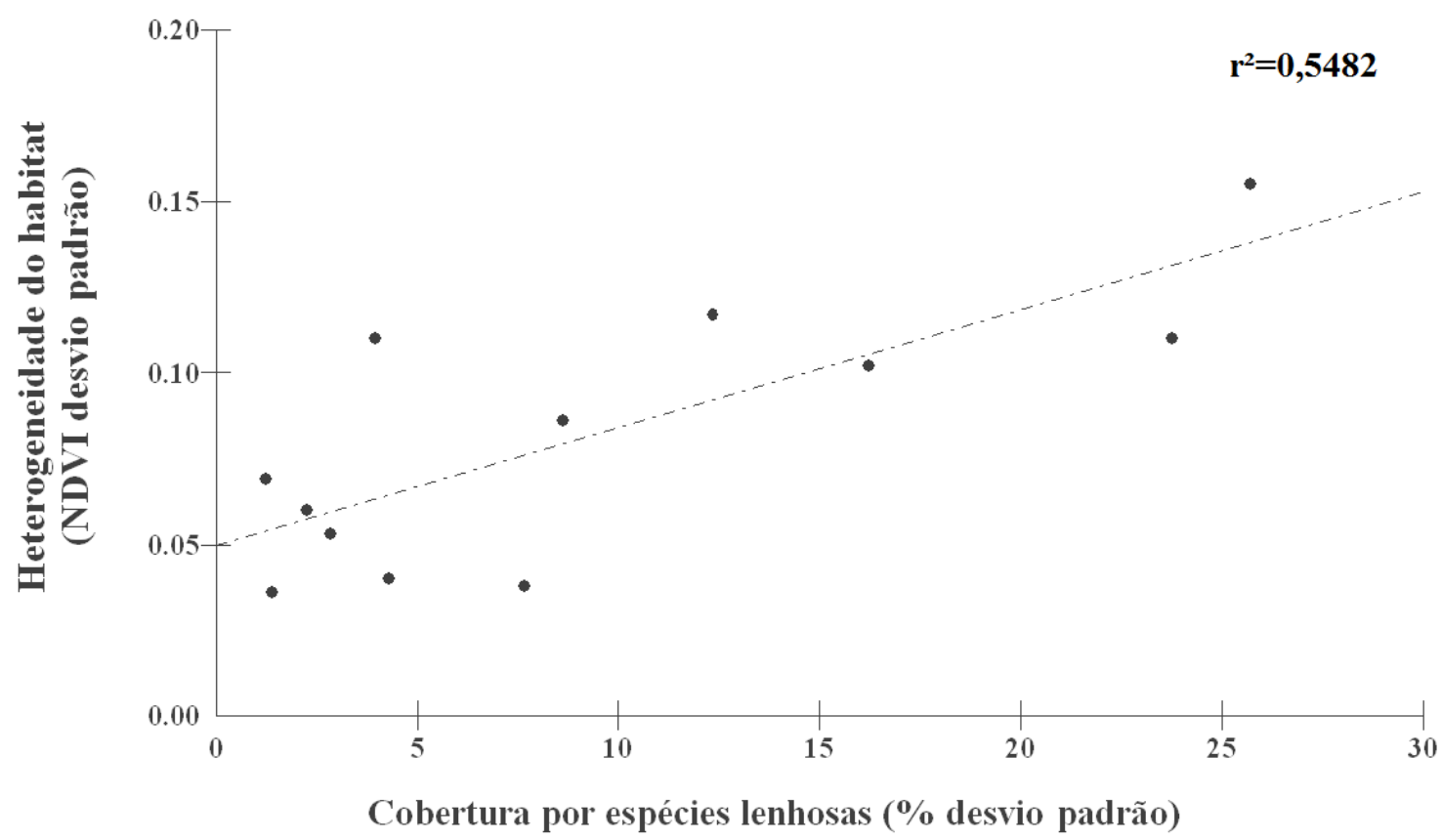

Figura 4. Relação entre heterogeneidade do habitat (NDVI desvio padrão) e cobertura por espécies lenhosas (\% desvio padrão).

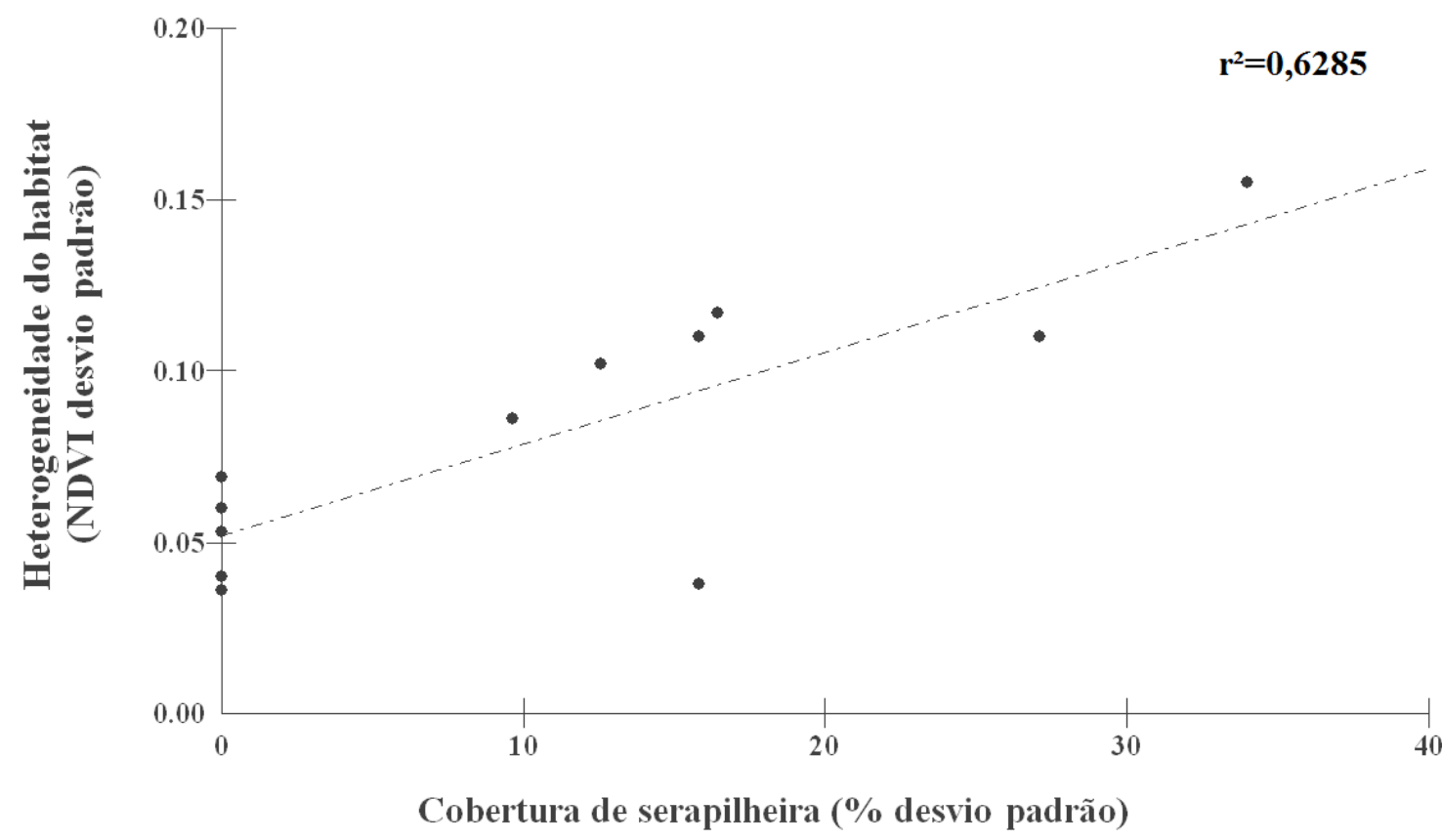

Figura 5. Relação entre heterogeneidade do habitat (NDVI desvio padrão) e cobertura de serapilheira (\% desvio padrão).

Outros estudos também relacionaram índice de vegetação com variáveis de estrutura da vegetação como Gamon et al. (1995, p. 28) em três tipos de 
vegetação nos Estados Unidos, Vicens et al. (2001, p. 32) na região de Floresta Atlântica de Tabuleiros, Giraldelli; Paranhos Filho (2006, p. 344) no Cerrado de Mato Grosso do Sul e Cândido et al. (2015, p. 101) no Cerrado de Mato Grosso.

O NDVI tem sido relacionado com a ocorrência de espécies de aves (SAVERAID et al., 2001, p. 71), riqueza de espécies de árvores tropicais (BAWA et al., 2002, p. 1), de aranhas (DE MAS et al., 2009, p. 1951), de besouros (LASSAU; HOCHULI, 2008, p. 139; CORRÊA et al., 2011, p. 3106) e com a abundância de flebotomíneos (OLIVEIRA et al., 2012, p. 397).

Foram analisadas as variações dos valores de NDVI de toda a área do PENRT para os anos de 1984, 1989, 1994, 1999, 2004 e 2010 (Tabela 2).

Tabela 2. Estatísticas calculadas a partir dos valores do NDVI de toda a área do PENRT em cada ano analisado.

\begin{tabular}{|c|c|c|c|c|}
\hline Ano & Média & $\begin{array}{l}\text { Desvio } \\
\text { padrão }\end{array}$ & Valor máximo & Valor mínimo \\
\hline $\mathbf{1 9 8 4}$ & 0,6941 & 0,2028 & 1 & -1 \\
\hline $\mathbf{1 9 8 9}$ & 0,7577 & 0,1963 & 1 & -1 \\
\hline $\mathbf{1 9 9 4}$ & 0,7430 & 0,1943 & 1 & -1 \\
\hline $\mathbf{1 9 9 9}$ & 0,6536 & 0,2418 & 1 & -1 \\
\hline $\mathbf{2 0 0 4}$ & 0,7581 & 0,2021 & 1 & -1 \\
\hline $\mathbf{2 0 1 0}$ & 0,6643 & 0,2410 & 1 & -1 \\
\hline
\end{tabular}

Os valores de complexidade do habitat (NDVI médio) para todo o PENRT oscilaram de 0,6536 a 0,7581, que correspondem a variação desde as formações savânicas até as formações campestres. Como se tratam de valores médios e o PENRT possui formações campestres nativas e exóticas, percebese que, as formações florestais contribuíram bastante na elevação desses valores.

Já os valores de heterogeneidade do habitat (NDVI desvio padrão) para todo o PENRT oscilaram de 0,1943 a 0,2418, indicando grande variação na 
estrutura vertical e da fitomassa do ambiente no plano horizontal, o que já era esperado visto que o PENRT é um mosaico de diferentes tipos de vegetação e cobertura do solo, com áreas sem vegetação como solo exposto e lagos, áreas úmidas, formações campestres nativas e exóticas, formações savânicas e florestais.

A complexidade do habitat (NDVI médio) para todo o PENRT no período de 1984 a 1989 aumentou 9,16\%. No período de 1989 a 1994 a complexidade do habitat do PENRT permaneceu praticamente a mesma, com uma pequena redução de 1,94\%. Já no período de 1994 a 1999 houve redução de 12,03\% na complexidade do habitat. No período de 1999 a 2004 houve aumento de quase 16\% na complexidade do habitat do PENRT. No período de 2004 a 2010 houve redução de $12,37 \%$ na complexidade do habitat.

Quando analisamos o período anterior à criação do PENRT, de 1984 a 1999 (ano da criação do Parque), percebemos uma redução de 5,83\% na complexidade do habitat do PENRT, indicativo de degradação das fitofisionomias arbóreas para formação de pastagem e extração de madeira ou também sofreram com incêndios, frequentes na região.

Já no período posterior à criação do PENRT, de 1999 a 2010, constatouse um pequeno aumento de $1,64 \%$ na complexidade do habitat, indicativo de regeneração das fitofisionomias arbóreas, provavelmente reflexo da proteção conferida pela criação da unidade de conservação.

Quando analisamos todo o período estudado, de 1984 a 2010, verificase pequena redução de 4,29\% na complexidade do habitat. A dinâmica da complexidade do habitat do PENRT ao longo de 26 anos (de 1984 a 2010) pode ser vista na Figura 6. 


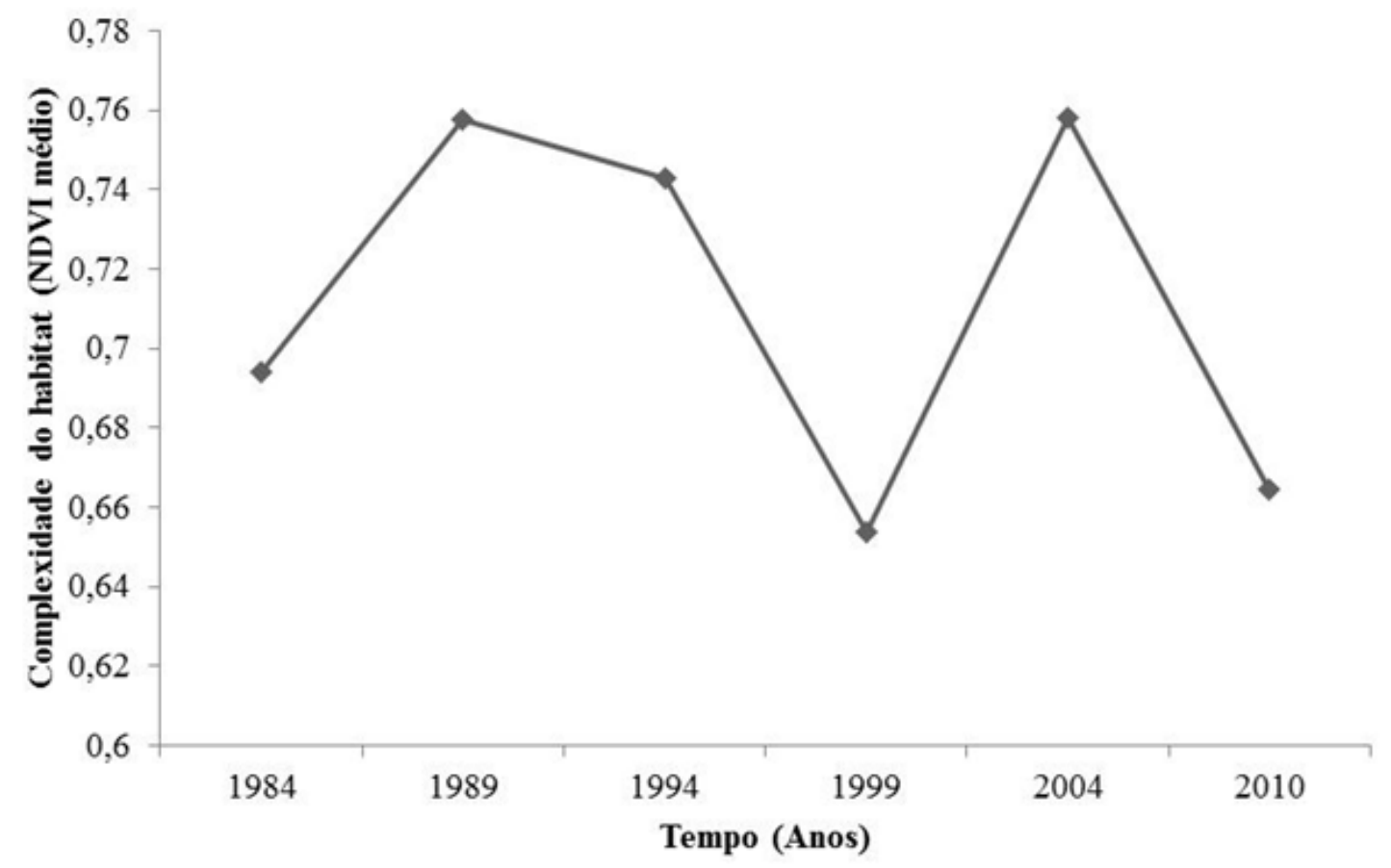

Figura 6. Análise multitemporal (dinâmica) da complexidade do habitat (NDVI médio) do PENRT ao longo de 26 anos (de 1984 a 2010).

Admite-se que a riqueza de espécies da floresta aumenta com a complexidade da estrutura vertical, visto que mais nichos estarão disponíveis (LANG; BLASCHKE, 2009, p. 302).

A heterogeneidade do habitat (NDVI desvio padrão) para todo o PENRT no período de 1984 a 1989 diminuiu 3,21\%. A redução na heterogeneidade do habitat indica uma simplificação no mosaico vegetacional, no caso do PENRT, pode ser que as fitofisionomias estejam evoluindo para um mesmo tipo de formação vegetal, de acordo com os dados de complexidade do habitat, para as formações savânicas e florestais.

No período de 1989 a 1994 houve pequena redução de 1,02\% na heterogeneidade do habitat. Já no período de 1994 a 1999 houve aumento de $24,45 \%$ na heterogeneidade do habitat. O aumento na heterogeneidade do habitat indica o crescimento no contraste do mosaico vegetacional que no caso do PENRT pode ser resposta da substituição das fitofisionomias arbóreas por outro tipo de cobertura do solo, ou seja, degradação de origem antrópica ou mesmo incêndios, frequentes na região. 
No período de 1999 a 2004 houve redução de 16,42\% na heterogeneidade do habitat do PENRT. Já no período de 2004 a 2010 houve aumento de 19,25\% na heterogeneidade do habitat.

Quando se analisa o período anterior à criação do PENRT, de 1984 a 1999 (ano da criação do Parque), percebe-se o aumento de 19,23\% na heterogeneidade do habitat do PENRT, indicativo de degradação.

Já no período posterior à criação do PENRT, de 1999 a 2010, a heterogeneidade do PENRT permaneceu praticamente a mesma, com uma pequena redução de $0,33 \%$, provavelmente reflexo da proteção conferida pela criação da unidade de conservação.

Quando analisamos todo o período estudado, de 1984 a 2010, verificase um aumento considerável de quase 19\% na heterogeneidade do habitat do PENRT, resposta ainda do período anterior à criação da unidade de conservação. A dinâmica da heterogeneidade do habitat do PENRT ao longo de 26 anos (de 1984 a 2010) pode ser vista na Figura 7.

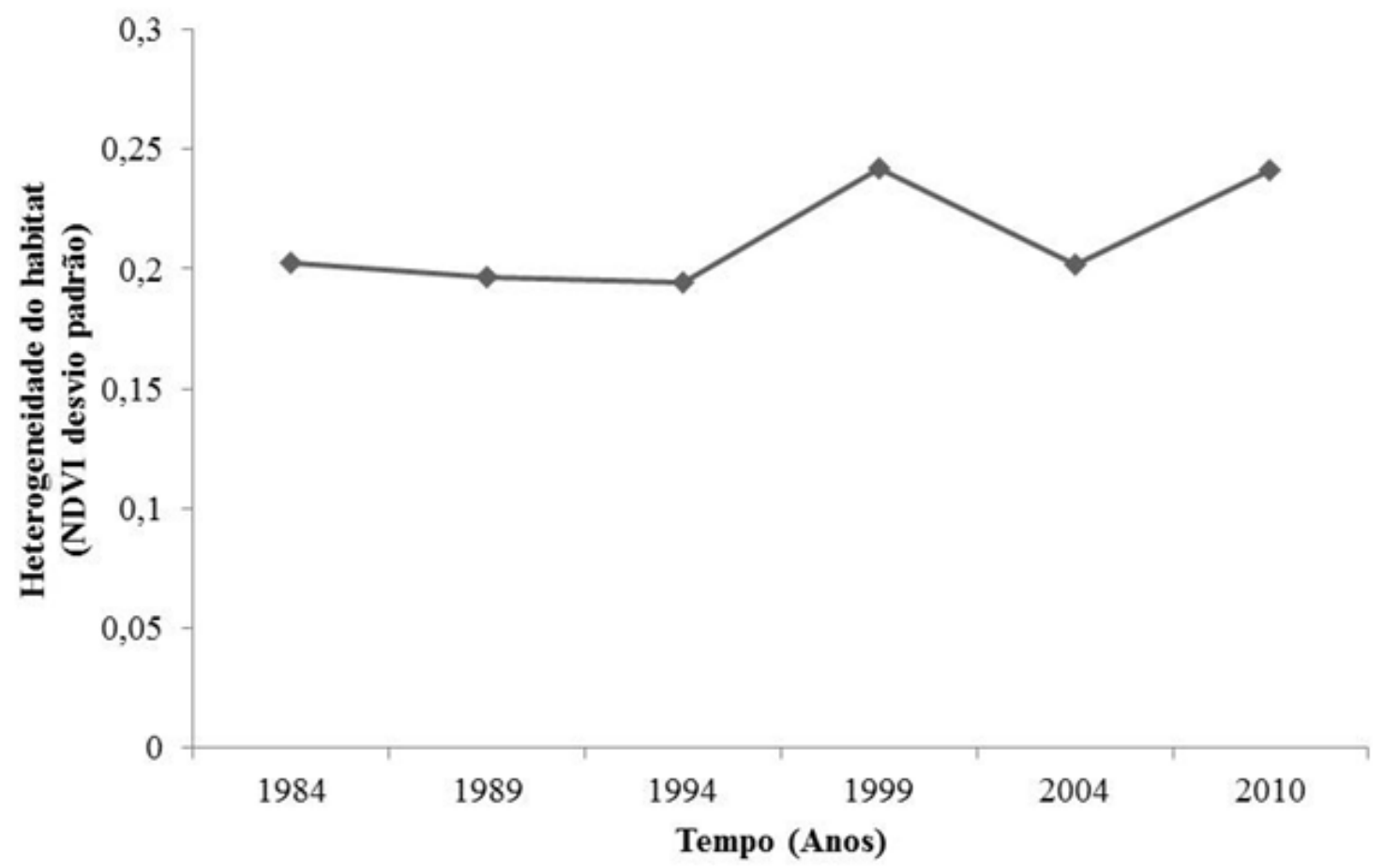

Figura 7. Análise multitemporal (dinâmica) da heterogeneidade do habitat (NDVI desvio padrão) do PENRT ao longo de 26 anos (de 1984 a 2010). 
A heterogeneidade ambiental é considerada um dos fatores determinantes da diversidade e distribuição de espécies. (SARMIENTO, 1984, p. 113).

Existe forte relação entre a diminuição da complexidade do habitat e o aumento da heterogeneidade para o PENRT como resultado de degradação das fitofisionomias arbóreas, tanto de origem antrópica como os incêndios que ocorrem naturalmente e são frequentes na região. No período de 1984 a 1989 houve aumento da complexidade e redução da heterogeneidade do habitat. Já no período de 1994 a 1999 percebe-se claramente grande redução na complexidade e expressivo aumento da heterogeneidade do habitat. No período de 1999 a 2004 a situação se inverteu, houve grande aumento da complexidade acompanhado da redução da heterogeneidade do habitat. No período de 2004 a 2010 a situação inverte-se novamente, houve grande redução na complexidade acompanhada do aumento na heterogeneidade do habitat. Essa evolução pode ser vista na Figura 8.

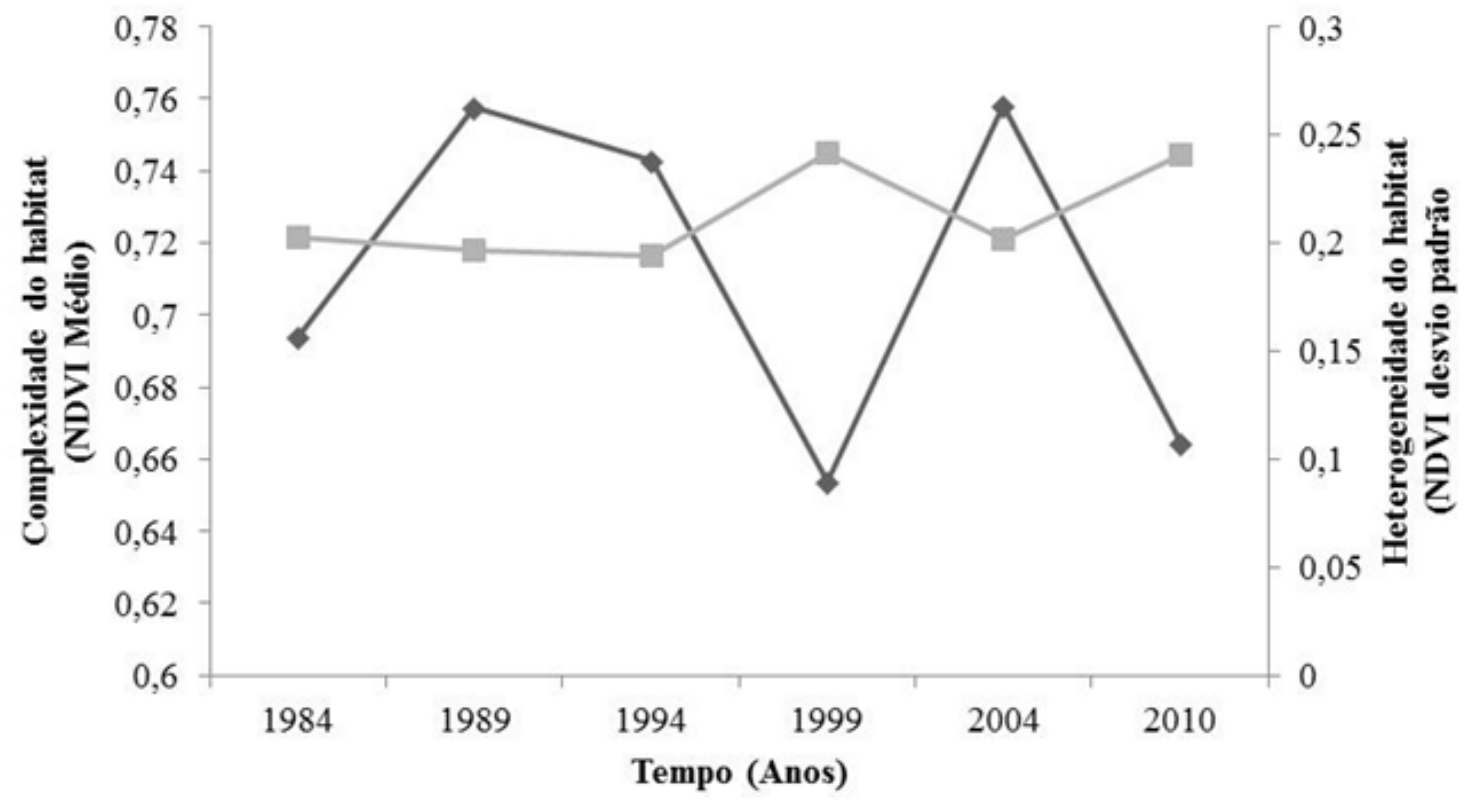

$\rightarrow$ Complexidade do habitat $\quad-\quad-$ Heterogeneidade do habitat

Figura 8. Análise multitemporal (dinâmica) da complexidade e heterogeneidade do habitat do PENRT ao longo de 26 anos (de 1984 a 2010).

Os componentes espaciais da paisagem, como a complexidade e heterogeneidade do habitat influenciam diretamente a diversidade $\mathrm{e}$ 
GAMARRA, R. M.; TEIXEIRA-GAMARRA, M. C.; CARRIJO, M. G. G.; PARANHOS FILHO, A. C. USO DO NDVI NA ANÁLISE DA ESTRUTURA DA VEGETAÇÃO E EFETIVIDADE DA PROTEÇÃO DE UNIDADE DE CONSERVAÇÃO NO CERRADO

distribuição de organismos, afetando variáveis como a presença e abundância das espécies e suas interações com o meio biótico (CHUST et al., 2004, p. 47; HIRAO et al., 2008, p. 192).

\section{CONCLUSÃO}

Conclui-se que a relação entre variáveis de estrutura da vegetação e o índice de vegetação (NDVI) e a análise multitemporal da complexidade e heterogeneidade do habitat mostraram-se ferramentas muito eficazes para avaliar a efetividade da proteção oferecida pela unidade de conservação. As geotecnologias contribuem para os estudos de ecologia e conservação da biodiversidade reduzindo tempo e custos na análise ambiental. Os métodos empregados no presente trabalho poderão ser utilizados em outras regiões do Cerrado que apresentam características semelhantes à área de estudo.

O PENRT é uma unidade de conservação nova, criada no ano de 1999 e grande parte da sua área ainda não foi desapropriada, assim ainda ocorrem atividades agropecuárias dentro do parque. Outra preocupação é sua zona de amortecimento, seu limite leste é totalmente tomado por extensas plantações de soja e algodão. Algumas nascentes (Ribeirão do Engano) encontram-se próximas ao parque, em seu entorno, mas fora da área de proteção, cercadas de plantações. Seria de grande importância que a região do entorno do parque também recebesse atenção, para que na medida em que as desapropriações forem sendo feitas, a vegetação do parque e seu entorno possa se regenerar.

\section{Agradecimentos}

Os autores externam seus agradecimentos à Fundação de Apoio ao Desenvolvimento do Ensino, Ciência e Tecnologia do Estado de Mato Grosso do Sul (FUNDECT-MS) pelos projetos de pesquisa $n^{\circ}$ 0081/08 e $n^{0}$ 0241/08 e pela bolsa de doutorado $n^{\circ}$ 057/10, ao Programa de Pós-Graduação em Ecologia e Conservação (PPGEC) da Universidade Federal de Mato Grosso do Sul (UFMS) e Coordenação de Aperfeiçoamento de Pessoal de Nível Superior (Capes) pela bolsa de doutorado, à SEMAC/IMASUL/GUC pela concessão da autorização ambiental para pesquisa em unidades de conservação $\mathrm{n}^{\circ}$ 
006/2008, ao seu Vanderlei e Professor Wilson (Prefeitura Municipal de Costa Rica) por todo apoio nos trabalhos de campo.

\section{FONTES DE FINANCIAMENTO}

Fundação de Apoio ao Desenvolvimento do Ensino, Ciência e Tecnologia do Estado de Mato Grosso do Sul (FUNDECT-MS): Projetos de pesquisa $\mathrm{n}^{\circ}$ 0081/08 e $n^{0}$ 0241/08 e bolsa de doutorado $n^{0}$ 057/10; Programa de PósGraduação em Ecologia e Conservação (PPGEC) da Universidade Federal de Mato Grosso do Sul (UFMS) e Coordenação de Aperfeiçoamento de Pessoal de Nível Superior (Capes): Bolsa de doutorado.

\section{REFERÊNCIAS}

ALHO, C.J.R.; MARTINS, E.S. De Grão em Grão o Cerrado Perde Espaço. (Cerrado - Impactos do Processo de Ocupação). WWF - Fundo Mundial para a Natureza, Brasília. 1995.

AYRES, M.; AYRES JUNIOR, M.; AYRES, D.L.; SANTOS, A.A.S.; AYRES, L.L. BioEstat versão 5.0 Aplicações Estatísticas nas Áreas das Ciências BioMédicas. Belém-PA. 2007.

BAWA, K.; ROSE, J.; GANESHAIAH, K.N.; BARVE, N.; KIRAN, M.C.; UMASHAANKER, R. Assessing biodiversity from space: an example from the Western Ghats, India. (http://www.ecologyandsociety.org/vol6/iss2/art7/). Acesso em 15 de julho de 2015. Conservation Ecology 6:7. 2002.

CÂNDIDO, A.K.A.A.; SILVA, N.M. da; BARBOSA, D.S. Dinâmica espacial e temporal do uso das terras e Índice de Vegetação por Diferença Normalizada (NDVI) em setor de cabeceira do rio São Lourenço, Campo Verde, MT. (http://ojs.c3sl.ufpr.br/ojs/index.php/raega/article/view/36338). Acesso em 15 de 
julho de 2015. Revista Ra'e Ga: O Espaço Geográfico em Análise. v. 33, p. 94-119. 2015.

CARVALHO, F.M.V.; MARCO JÚNIOR, P.; FERREIRA, L.G. The Cerrado intopieces: Habitat fragmentation as a function of landscape use in the savannas of central

Brazil. (http://www.sciencedirect.com/science/article/pii/S000632070900072X). Acesso em 15 de julho de 2015. Biological Conservation. 142. 1392-1403. 2009.

CHUST, G.; PRETUS, J.L.; DUCROT, D.; VENTURA, D. Scale dependency of insect assemblages in response to landscape pattern. (http://link.springer.com/article/10.1023\%2FB\%3ALAND.0000018368.99833.f2\# /page-1). Acesso em 15 de julho de 2015. Landscape Ecology 19: 41-57. 2004.

CONSERVATION INTERNATIONAL DO BRASIL. Corredor de Biodiversidade Emas-Taquari. Disponível em: (http://www.conservation.org.br/onde/cerrado/index.php?id=155). Acesso em: 18 de setembro de 2014. 2003.

CORRÊA, C.C.; PIMENTA, M.; DUTRA, S.L.; MARCO JÚNIOR, P. Utilização do NDVI na avaliação da resposta de besouros herbívoros à complexidade e heterogeneidade ambiental em diferentes escalas no Bioma cerrado. In: Anais XV SIMPÓSIO BRASILEIRO DE SENSORIAMENTO REMOTO, INPE, Curitiba, p. 3103-3110. 2011.

DE MAS, E.; CHUST, G.; PRETUS, J.L.; RIBERA, C. Spatial modelling of spider biodiversity: matters of scale. (http://link.springer.com/article/10.1007\%2Fs10531-008-9566-2\#/page-1). Acesso em 15 de julho de 2015. Biodiversity and Conservation 18:19451962. 2009. 
FELFILI, J.M. Padrões de diversidade do Cerrado do Centro-Oeste Brasileiro. In: ARAÚJO, E.L.; MOURA, A.N.; SAMPAIO, E.S.B.; GESTINARI, L.M.S \& CARNEIRO, J.M.T. (Org.). Biodiversidade, Conservação e Uso Sustentável da Flora do Brasil. Recife-PE: UFRPE-Imprensa Universitária, 2002. Capitulo 5, 58-61.

FELFILI, J.M.; NOGUEIRA, P.E; SILVA-JÚNIOR, M.C.; MARIMOM, B.S.; DELITTI, W. B. C. Composição florística e fitossociologia do cerrado sentido restrito no município de Água Boa - MT. (http://www.scielo.br/scielo.php?script=sci_arttext\&pid=S010233062002000100012). Acesso em 15 de julho de 2015. Acta Botanica Brasilica. 16(1): 103-112. 2002.

FORMAN, R.T.; GODRON, M. Landscape Ecology. John Willey. Nova lorque. 619 p. 1986.

GAMARRA, R.M. Geotecnologias na análise da estrutura e dinâmica da paisagem do Parque Estadual das Nascentes do rio Taquari-MS. 2013. Tese (Doutorado em Ecologia e Conservação). Centro de Ciências Biológicas e da Saúde (CCBS), Universidade Federal de Mato Grosso do Sul (UFMS), Campo Grande-MS.

GAMON, J.A.; FIELD, C.B.; GOULDEN, M.L.; GRIFFIN, K.L.; HARTLEY, A.E.; JOEL, G.; PEÑUELAS, J.; VALENTINI, R. Relationships between NDVI, canopy structure, and photosynthesis in three Californian vegetation types. (http://escholarship.org/uc/item/86c8v3bf\#page-3). Acesso em 15 de julho de 2015. Ecological Applications, 5(1):28-41.1995.

GEWIN, V. Mapping opportunities. Nature 427. p. 376-377. 22 January 2004.

GIRALDELLI, G.R.; PARANHOS FILHO, A.C. Relação entre as variáveis estruturais do habitat e o índice de vegetação de diferença normalizada (NDVI) 
em três tipos de habitat de cerrado na fazenda Diamante, Coxim, MS. Anais $1^{\circ}$ SIMPÓSIO DE GEOTECNOLOGIAS NO PANTANAL, Campo Grande, Mato Grosso do Sul, Brasil. Embrapa Informática Agropecuária/INPE, p.341-350. 2006.

GLOBALGEO. Globalgeo Geotecnologias LTDA. Imagem do satélite ALOS, sensor AVNIR-2. Bandas 1, 2, 3 e 4. ID ALAV2A236673960. Órbita/Ponto 405/3960 de 04 de julho de 2010. 2010 a.

GLOBALGEO. Globalgeo Geotecnologias LTDA. Imagem do satélite ALOS, sensor PRISM. Banda Pan. ID ALPSMN243383960. Órbita/Ponto 405/3960 de 19 de agosto de 2010. 2010 b.

HIRAO, T.; MURAKAMI, M.; IWAMOTO, J.; TAKAFUMI, H.; OGUMA, H. Scaledependent effects of windthrow disturbance on forest arthropod communities. (http://link.springer.com/article/10.1007\%2Fs11284-007-0370-3\#/page-1). Acesso em 15 de julho de 2015. Ecological Research 23: 189-196. 2008.

INPE. Instituto Nacional de Pesquisas Espaciais. Imagem do satélite LANDSAT 5, sensor TM. Bandas 1, 2, 3, 4, 5, 6 e 7. Órbita/Ponto 224/073 de 18 de julho de 1984. Disponível em: (http://www.dgi.inpe.br/CDSR/). Acesso em: 12 de novembro de 2012. 1984.

INPE. Instituto Nacional de Pesquisas Espaciais. Imagem do satélite LANDSAT 5, sensor TM. Bandas 1, 2, 3, 4, 5, 6 e 7. Órbita/Ponto 224/073 de 16 de julho de 1989. Disponível em: (http://www.dgi.inpe.br/CDSR/). Acesso em: 12 de novembro de 2012. 1989.

INPE. Instituto Nacional de Pesquisas Espaciais. Imagem do satélite LANDSAT 5, sensor TM. Bandas 1, 2, 3, 4, 5, 6 e 7. Órbita/Ponto 224/073 de 30 de julho de 1994. Disponível em: (http://www.dgi.inpe.br/CDSR/). Acesso em: 12 de novembro de 2012. 1994. 
INPE. Instituto Nacional de Pesquisas Espaciais. Imagem do satélite LANDSAT 5, sensor TM. Bandas 1, 2, 3, 4, 5, 6 e 7. Órbita/Ponto 224/073 de 28 de julho de 1999. Disponível em: (http://www.dgi.inpe.br/CDSR/). Acesso em: 12 de novembro de 2012. 1999.

INPE. Instituto Nacional de Pesquisas Espaciais. Imagem do satélite LANDSAT 5, sensor TM. Bandas 1, 2, 3, 4, 5, 6 e 7. Órbita/Ponto 224/073 de 25 de julho de 2004. Disponível em: (http://www.dgi.inpe.br/CDSR/). Acesso em: 12 de novembro de 2012. 2004.

INPE. Instituto Nacional de Pesquisas Espaciais. Imagem do satélite LANDSAT 5, sensor TM. Bandas 1, 2, 3, 4, 5, 6 e 7. Órbita/Ponto 224/073 de 26 de julho de 2010. Disponível em: (http://www.dgi.inpe.br/CDSR/). Acesso em: 12 de novembro de 2012. 2010.

LANG, S.; BLASCHKE, T. Análise da paisagem com SIG. Tradução Hermann Kux. São Paulo-SP: Oficina de Textos, 2009.

LASSAU, S.A.; HOCHULI, D.F. Testing predictions of beetle community patterns derived empirically using remote sensing. Diversity and Distributions 14:138-147. 2008.

LIU, W.T.H. Aplicações de Sensoriamento Remoto. Campo Grande: Editora UNIDERP, 2007.

MACHADO, R.B.; RAMOS NETO, M.B.; PEREIRA, P.G.P.; CALDAS, E.F.; GONÇALVES, D.A.; SANTOS, N.S.; TABOR, K.; STEININGER, M. Estimativas de perda da área do Cerrado brasileiro. Relatório técnico não publicado. Conservação Internacional, Brasília, DF. 2004. 
MATO GROSSO DO SUL. Decreto no 9.662, de 9 de outubro de 1999. Cria o Parque Estadual das Nascentes do Rio Taquari, e dá outras providências. Publicado no Diário Oficial de Mato Grosso do Sul de 14 de outubro de 1999.

MitTeRMeieR, R. A.; MYERS, N.; GIL, P. R.; MitTERMEIER, C. G. Hotspots: Earth's Biologically Richest and Most Endangered Terrestrial Ecoregions. Conservation International and Agrupacion Sierra Madre (Cemex), Monterrey, Mexico, 1999.

MMA. Ministério do Meio Ambiente. Áreas Prioritárias para Conservação, Uso Sustentável e Repartição de Benefícios da Biodiversidade Brasileira: atualização Portaria $n^{\circ}$ 9, de 23 de janeiro de 2007. Brasília: MMA, Secretaria de Biodiversidade e Florestas. 2007.

OLIVEIRA, E. F.; SILVA, E. A.; FERNANDES, C. E.; PARANHOS FILHO, A.C.; GAMARRA, R. M.; RIBEIRO, A. A.; BRAZIL, R.P.; OLIVEIRA, A.G. Biotic factors and occurrence of Lutzomyia longipalpis in endemic area of visceral leishmaniasis, Mato Grosso do Sul, Brazil. Memórias do Instituto Oswaldo Cruz (Impresso). 2012.

PARANHOS FILHO, A.C.; LASTORIA, G.; TORRES, T.G. Sensoriamento Remoto Ambiental Aplicado: Introdução as Geotecnologias. Campo Grande-MS: Editora UFMS, 2008.

PCI. Geomatica Versão 9.1 for Windows. Ontário, Canadá. 16 de dezembro de 2003. 1 CD-ROM. 2003.

PONZONI F.J.; SHIMABUKURO Y.E. Sensoriamento Remoto no Estudo da Vegetação. São José dos Campos-SP: INPE-Editora Parêntese, 2007. 
RIBEIRO, J.F.; WALTER, B.M.T. Fitofisionomias do Bioma Cerrado. In. SANO, S.M. \& ALMEIDA, S.P. Cerrado: ambiente e flora. Planaltina-DF: EMBRAPACPAC, 1998. Capítulo 3, 87-165.

ROOT, R.B. Organization of a plant-arthropod association in a simple and diverse habitats: the fauna of collards (Brassica oleracea). Ecological Monographs 43:95-124. 1973.

ROUGHGARDEN, J.; RUNNING, S.W.; MATSON, P.A. What does Remote Sensing do for Ecology?. Ecology. 72(6). pp 1918-1922. 1991.

ROUSE, J.W.; HAAS, R.H.; SCHELL, J.A.; DEEERING, D.W. Monitoring vegetation systems in the Great Plains with ERTS (Earth Resources Technology Satellite). In: PROCEEDINGS OF THE THIRD ERTS SYMPOSIUM, SP-351 Goddard Space Flight Center, 1973, Washington: NASA, 1973, p. 309-317.

SARMIENTO, G. The ecology of Neotropical savannas. Harvard University Press, Cambridge, 256 pp. 1984.

SAVERAID, E.H.; DEBINSKI, D.M.; KINDSCHER, K.; JAKUBAUSKAS, M.E. A comparison of satellite data and landscape variables in predicting bird species occurrences in the Greater Yellowstone Ecosystem, USA. (http://link.springer.com/article/10.1023\%2FA\%3A1008119219788\#page-1). Acesso em 15 de julho de 2015. Landscape Ecology 16:71-83. 2001.

SISLA. Sistema Interativo de Suporte ao Licenciamento Ambiental. (http://sisla.imasul.ms.gov.br/Downloads/dados_complementares/). Último acesso em 5 de novembro de 2012. Instituto de Meio Ambiente de Mato Grosso do Sul (IMASUL). 2008. 
TURNER, M.G. Spatial simulation of landscape changes in Georgia: a comparison of 3 transition models. Landscape Ecology. 1: 27-39. 1987.

VICENS, R. S.; MARQUES, J. S.; CRUZ, C.B.M.; ARGENTO, M. F.; GARAY, I. Sensoriamento Remoto e SIG como suporte ao desenvolvimento do subprojeto PROBIO "Conservação e recuperação da Floresta Atlântica de Tabuleiros". In: GARAY, I. \& DIAS, B.F.S. (Orgs.). Conservação da Biodiversidade em Ecossistemas Tropicais. Rio de Janeiro-RJ: Editora Vozes, 2001. Capítulo 12, 317-337. 